\title{
Metabolomics of potential contenders conferring antioxidant property to varied polar and non-polar solvent extracts of Edgaria darjeelingensis C.B.Clarke
}

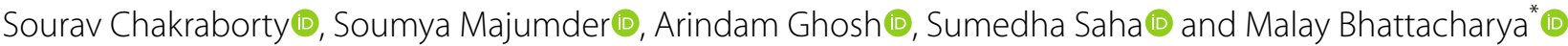

\begin{abstract}
Background: The Himalaya hotspot, habitat to numerous indigenous species, harbours an endemic and endangered, cucurbit climber Herpetospermum darjeelingense (C.B.Clarke) H.Schaef. \& S.S.Renner [Edgaria darjeelingensis C.B.Clarke]. Seeds of this plant have traditionally been used for ethno-veterinary treatment. The present probe aims to provide an insight into the antioxidant-related biochemical attributes of this phytochemically unexplored plant.

Results: DPPH scavenging activity of solvent extracts showed more potential towards the polar end with its maximum activity in methanolic extract (84.64\%). Acetone extract showed more ferric reducing power than the ethanol fractions. Maximum flavonoid quantification was recorded in ethanol extract. GC-MS of methanolic extract detected 39 compounds of which 22 have preventive and curing properties for several ailments. Thirteen compounds were reported with antioxidant activity and cover an area of $63.92 \%$ among the total detected compounds by GC-MS. 1,3,4,5-Tetrahydroxy-cyclohexanecarboxylic acid; Hexadecanoic acid, methyl ester; $ß$ sitosterol and Phytol are present in significant quantity and are the prime candidates conferring antioxidant potential. Chromatographically fractionalized methanol extract with non-polar to polar solvents and their subsequent DPPH scavenging analysis exhibited a major peak in ethanol fraction due to the presence of Phytol in considerable high quantity and some other compounds. Further sub-fractionation of ethanolic fraction provides a supportive evidence of ethanol solubility of the compounds. Biosynthetic lineage reveals fatty acid synthesis, intermediates of Shikimate pathway and terpenoid metabologenesis schemes lead to the several antioxidant molecules detected in our extract.
\end{abstract}

Conclusion: Metabolomics of antioxidants will provide a better understanding in its intracellular free radical quenching activity and isolation of these bioactive compounds can boost pharmaceutical industry.

Keywords: Edgaria darjeelingensis, Endemic, Endangered, Antioxidant, GC-MS, Pathway

\section{Background}

India is a mega-diversity nation owing to its rich floral and faunal variations. It harbours several hotspots of which the Himalaya Hotspot is habitat to numerous endemic and indigenous species. The Eastern Himalaya

\footnotetext{
*Correspondence: malaytsnbu@gmail.com

Molecular Biology and Tissue Culture Laboratory, Department of Tea

Science, University of North Bengal, Siliguri, Darjeeling, West Bengal 734013, India
}

including Darjeeling hills (average $6700 \mathrm{ft}$ ) shares boundaries with Eastern Nepal in the west and Bhutan and Sikkim in the North East and thus can be considered as a typical geographical terrain assembling and uniting the various endemic as well as exotic flora of India, Bhutan and Nepal. Out of the various endemic species and among the $11.61 \%$ indigenous climbers of the Darjeeling Eastern Himalayas (https://shodhganga inflibnet.ac.in/bitstream/10603/147123/14/14_chapt er_09); a very phytochemically active but biochemically 
under investigated endemic Cucurbit climber is Edgaria darjeelingensis C.B.Clarke [Herpetospermum darjeelingense (C.B.Clarke) H. Schaef. \& S.S. Renner]. The plant is not only endemic but is also enlisted under the endangered category confronting a very high risk of extinction in the wild in the near future as per scientific records. The angiospermic family Cucurbitaceae ranks second (only next to Papilionaceae) among the climber dominating families in the Darjeeling hills of Eastern Himalaya with about twenty-seven reported species (https://shodhganga.inflibnet.ac.in/bitstream/10603 /147123/14/14_chapter_09).

The referred plant occurs at an altitude of 1450$3000 \mathrm{~m}$ and is a climber characterized by axillary shoot tendril where axillary buds are modified into tendrils for climbing purposes. E. darjeelingensis is prevalently known as Cathil in the Chitwan district of Nepal and its fruits are economically useful as a source of vegetable to the local people specifically the ancient tribal ethnic group 'Tharu' (Mueller-Boeker 1993). The 'Tamang' community in Central Nepal and Darjeeling Eastern Himalayas also employs E. darjeelingensis in herbal veterinary medications where it is commonly known as 'Tangsarkato' among the Tamang's and 'Jangali Karela' among the local Nepali inhabitants. The seeds of the plant are mentioned to be used for bovine treatment as per the ethno medicinal records of the 'Tamang' community. The methodology involves the mixing of the pounded seeds with corn flour and is fed to the cattle for relief from fever (Shrestha and Khadgi 2019). Thus, E. darjeelingensis is an endemic, endangered and ethno-medicinally important climber Cucurbit of Darjeeling Eastern Himalaya hotspot that has remained neglected from the biochemical and molecular characterizations till date. The present probe aims to explore and unveil the various antioxidant-related phytochemical attributes of the under explored Cucurbit climber.

\section{Methods}

\section{Collection of plant material}

Plant materials [leaves of E.darjeelingensis (ED)] were collected from the junction of Lebong Cart Road and Happy Valley Tea Estate, Darjeeling (27.050904 $\mathrm{N}$ and $88.260809^{\circ} \mathrm{E}$ ). The plant was field identified by Senior Taxonomist of Darjeeling Government College, Darjeeling. Since this plant is an endangered species, utmost care was taken during sample collection so as to cause minimal injury to the plant body. Vegetative leaves were detached from mature shoots, filled in zipper bags and preserved in insulated ice packed boxes for transference to laboratory.

\section{Sample extracts preparation}

ED leaves were washed thoroughly in running tap water to remove any adherent dust particles followed by final cleansing with distilled water. Wet leaves were then surface-dried using tissue paper and mechanically grinded to obtain a powdery mass. $3 \mathrm{~g}$ of powdered leaf sample was measured in a digital balance and poured in each of the reaction tubes containing a range of non-polar to polar solvents (hexane, toluene, chloroform, diethyl ether, ethyl acetate, acetone, ethanol, methanol and water). Reaction mixtures were allowed to stand for $48 \mathrm{~h}$ at room temperature after which the extracts were filtered through ash-less filter paper. Next the respective solvents in the reaction tubes were made to completely dry out at a mild temperature and finally the remnants were dissolved in methanol adjusting the concentration to $0.5 \mathrm{~g} / \mathrm{ml}$. For GC-MS analysis, the methanolic extract was adjusted at a concentration of $25 \mathrm{mg} / \mathrm{ml}$.

\section{Assay of antioxidant activity DPPH induced free radical scavenging assay}

The protocol of Bhattacharya et al. (2009) was followed for analysing free radical scavenging activity through DPPH assay technique. $0.3 \mathrm{ml}$ ED leaf extract was added to $2 \mathrm{ml}$ methanolic solution of $0.2 \mathrm{mM} \mathrm{DPPH}$ (2,2-diphenyl-1-picrylhydrazyl). The reaction mixture was vortexed vigorously and incubated at room temperature for 30 minutes in dark. Absorbance was measured at $517 \mathrm{~nm}$ using UV-Vis spectrophotometer and ascorbic acid $(\mu \mathrm{g} /$ $\mathrm{ml}$ methanol) was taken as standard. DPPH scavenging percentage was calculated as follows:

$$
\begin{aligned}
& \text { Free radical scavenging activity }(\%) \\
& \qquad=\left(A_{\text {Control }}-A_{\text {Sample }}\right) \times 100 / A_{\text {Control }}
\end{aligned}
$$

where $A_{\text {Control }}=$ absorbance value of Control and $A_{\text {Sam- }}$ ple $=$ Absorbance value of Sample. Ascorbic acid equivalent of potential DPPH scavenger was calculated from the standard curve.

The study of interaction and DPPH inhibition kinetics of ED leaf extracts in acetone, ethanol and methanol were conducted at $517 \mathrm{~nm} .0 .3 \mathrm{ml}$ ED leaf extract was added to $2 \mathrm{ml}$ methanolic solution of $0.2 \mathrm{mM} \mathrm{DPPH}$ in a quartz cuvette and placed in UV-Vis spectrophotometer for 30 minutes. Absorbance kinetics obtained from the graph was used to determine the time required for $50 \%$ scavenging activity.

\section{Ferric reducing power (FRP) assay of potential non-polar to polar solvent extracts}

Ferric reducing power (FRP) assay was performed following the protocol of Labar et al. (2019). To $200 \mu \mathrm{l}$ of 
ED leaf extract, $500 \mu \mathrm{l}$ phosphate buffer ( $\mathrm{pH} 7.4$ ) was added followed by addition of $1 \% 500 \mu$ l potassium ferricyanide $\left[\mathrm{K}_{3} \mathrm{Fe}(\mathrm{CN})_{6}\right]$ solution The reaction mixture was incubated for 20 minutes at $50{ }^{\circ} \mathrm{C}$, after which $10 \%$ $500 \mu \mathrm{l}$ trichloroacetic acid, $1.5 \mathrm{ml}$ distilled water and $0.1 \%$ $(\mathrm{w} / \mathrm{v}) 300 \mu \mathrm{l}$ ferric chloride $\left(\mathrm{FeCl}_{3}\right)$ solution was added. The absorbance was measured at $700 \mathrm{~nm}$ by an UV-Vis spectrophotometer.

\section{Flavonoid quantification}

To $250 \mu \mathrm{l}$ of ED leaf extract in a test tube, $1.25 \mathrm{ml}$ double distilled water and $75 \mu \mathrm{l} 5 \% \mathrm{NaNO}_{2}$ (sodium nitrite) were added and allowed to stand at room temperature for 5 minutes. Then, $150 \mu \mathrm{l} 10 \% \mathrm{AlCl}_{3}$ (Aluminium chloride) was added to the reaction mixture and kept stationary for 6 minutes at room temperature. After that $500 \mu \mathrm{l}$ of $1 \mathrm{mM}$ sodium hydroxide $(\mathrm{NaOH})$ and $275 \mu \mathrm{l}$ of double distilled water were subsequently added and the solution was incubated for 30 minutes. Finally, the absorbance was measured at $510 \mathrm{~nm}$ utilizing UV-Vis spectrophotometer (Zou et al. 2004).

\section{Gas chromatography-mass spectrometry analysis}

GC-MS analysis was conducted following the protocol of Majumder et al. (2020). Methanol extract of ED (25 mg/ $\mathrm{ml}$ ) was used for GC-MS. $1 \mu \mathrm{l}$ of extract was injected in split mode in the instrument (GCMS-QP2010 Plus). Injection temperature $\left(260{ }^{\circ} \mathrm{C}\right)$ and interface temperature $\left(270{ }^{\circ} \mathrm{C}\right)$ were maintained. Ion Source temperature was adjusted to $230{ }^{\circ} \mathrm{C}$. Helium was used as carrier gas. Total flow rate was $16.3 \mathrm{ml} \mathrm{min}{ }^{-1}$ and column flow rate was $1.21 \mathrm{ml} \mathrm{min}^{-1}$. Mass spectra were recorded at 5 scan $\mathrm{sec}^{-1}$ with a scanning range of $40-650 \mathrm{~m} / \mathrm{z}$. Quantification of compounds was done on the basis of their peak areas. The data obtained from GCMS analysis were further analysed through the available literature.

\section{Chromatographic separation and DPPH scavenging assay}

Bioactive molecules conferring antioxidant potential differ by their preference for solvent. So, the extract in methanol was fractionized by solvents like toluene, chloroform, diethyl ether, ethanol, ethanol/methanol (1:1), methanol, methanol/water (1:1) and water to obtain their respective fractions. A part of the ethanol fraction was further fractionated by ethanol in a silica column and were collected as $10 \mathrm{ml}$ fractions. DPPH scavenging ability for each fraction was conducted and calculations were made in accordance with previously described protocol (Bhattacharya et al. 2009).

Biosynthesis pathway of potential antioxidant compounds Biosynthesis pathways of some detected antioxidant molecules detected in GC-MS were done by reviewing several literatures and databases like PubChem (https ://pubchem.ncbi.nlm.nih.gov), UniProt (https://www. uniprot.org/) and KEGG pathway (https://www.genom e.jp/kegg/pathway.html).

\section{Statistical analysis}

Statistical analysis, viz. mean and standard deviation, was calculated on the basis of results obtained from three replications. Ascorbic acid $(\mu \mathrm{g} \mathrm{AE} / \mathrm{ml})$ and quercetin (mg QE/g) equivalent were calculated based on the mean value. Standard error bars are provided in graphical representation of data.

\section{Results \\ Antioxidant activity assay DPPH induced free radical scavenging assay}

Non-polar to polar solvent extracts of ED showed variable ability to scavenge $\mathrm{DPPH}$ exhibiting an ample range of free radical scavenging activity (Fig. 1a). The percentage of recorded DPPH activity in solvent extracts showed an increase towards the polar end (Hexane-12.75 \pm 0.385072 ; Toluene-12.66 \pm 0.326103 ; Chloroform $-26.64 \pm 0.421570$; Diethyl ether-14.30 \pm 0.297658 ; Ethyl acetate-27.68 $\pm 0.394356 ; \quad$ Acetone $-26.23 \pm 0.448723$; Ethanol-54.22 $\pm 0.573204 ;$ Water-M-23.27 \pm 0.386675 and Water-W-36.71 \pm 0.297042$)$ with its maximum activity in methanolic extract $(84.64 \pm 0.335012)$. Ascorbic acid equivalent (AE) calculated from the standard curve, $y=0.876 x+6.591 \quad\left(R^{2}=0.993\right)$ for $\mathrm{DPPH}$ scavenging activity of methanol extract, was found to be $89.005 \mu \mathrm{g} \mathrm{AE} / \mathrm{ml}$. Water extract was divided into two parts-methanol soluble (Water-M) and water soluble (Water-W) parts. Part that was insoluble in methanol but soluble in water (Water-W) exhibited more DPPH scavenging property. Solvent extracts of hexane, toluene, chloroform, diethyl ether and ethyl acetate showed DPPH scavenging activity below $40 \%$. Extracts with acetone and ethanol exhibited moderate activity while methanol showed maximum potential in scavenging DPPH. Interaction and DPPH inhibition kinetics of ED leaf extracts in acetone, ethanol and methanol are depicted in Fig. 1b-d. Graphical representation of absorbance kinetics in acetone extract portrayed consecutive rise and fall in DPPH scavenging activity while a steady increase in DPPH quenching activity was recorded in ethanol and methanol extracts, respectively. The time taken for $50 \%$ scavenging activity was minimum in acetone extract (2.9minute) followed by ethanol (5.2minute) and methanol (7.5minute). 


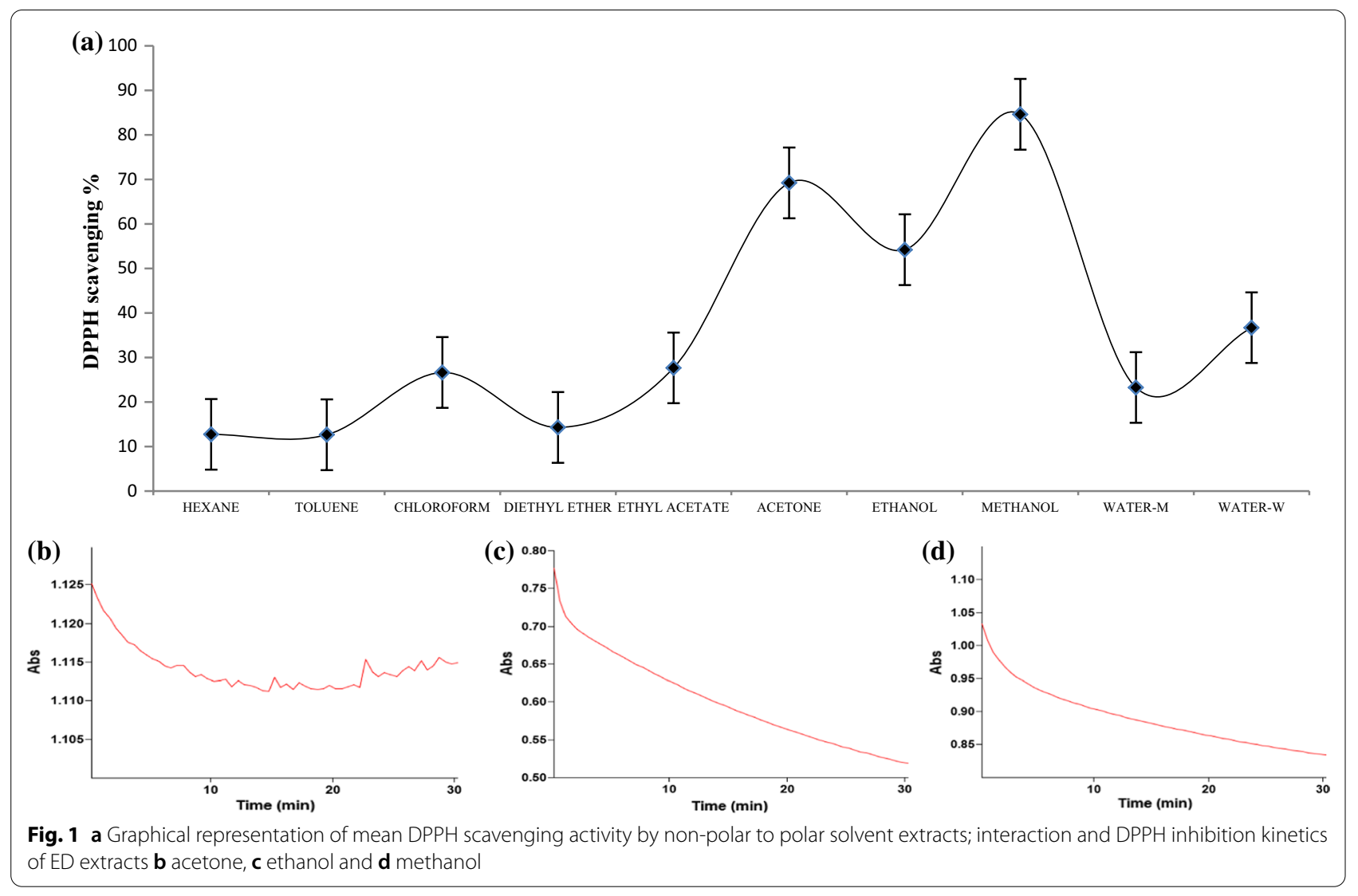

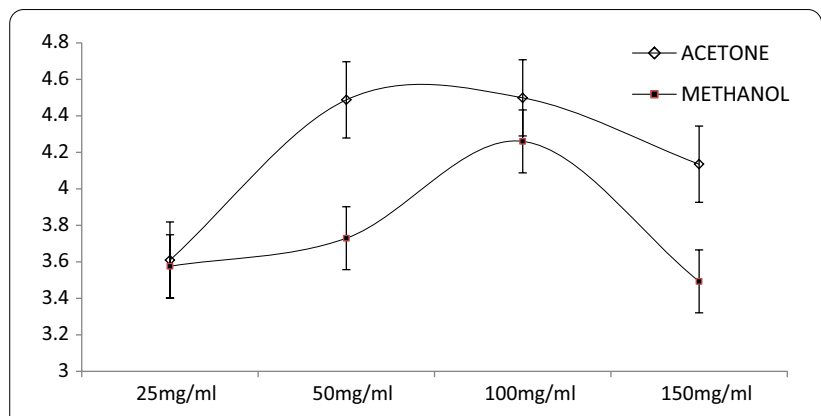

Fig. 2 Graphical representation of mean ferric reducing power assay of potential non-polar to polar solvent extracts

\section{Ferric reducing power (FRP) assay of potential non-polar to polar solvent extracts}

Ferric reducing power (FRP) assay was performed by four concentrations $(50,100,200$ and $300 \mathrm{mg} / \mathrm{ml}$ ) to assess the influence of molecular quantity in reducing ferric ions. ED leaf extract showed increase in FRP gradually from $50 \mathrm{mg} / \mathrm{ml}$ to $200 \mathrm{mg} / \mathrm{ml}$ (Acetone fraction: $50 \mathrm{mg} /$ $\mathrm{ml}-3.6097 \pm 0.425621,100 \mathrm{mg} / \mathrm{ml}-4.4878 \pm 0.362904$, $200 \mathrm{mg} / \mathrm{ml}-4.4988 \pm 0.393742$ and $300 \mathrm{mg} /$ ml-4.1353 \pm 0.479311 ; Methanol fraction: $50 \mathrm{mg} /$ $\mathrm{ml}-3.5761 \pm 0.287643,100 \mathrm{mg} / \mathrm{ml}-3.7298 \pm 0.457201$,

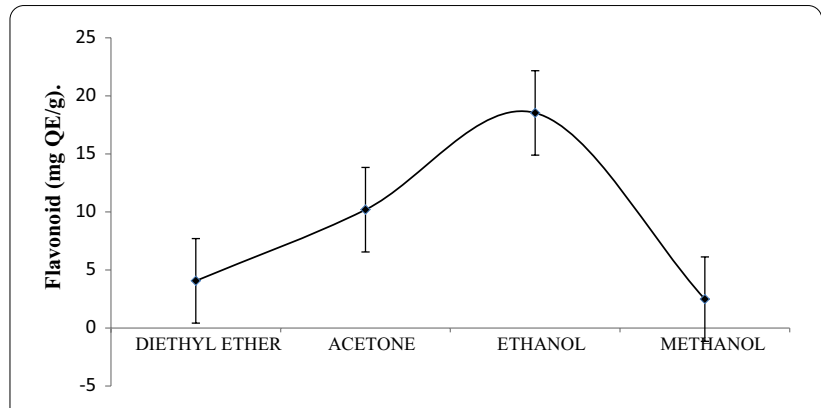

Fig. 3 Graphical representation of mean flavonoid content expressed as quercetin equivalent ( $\mathrm{mg} \mathrm{QE} / \mathrm{g}$ )

$200 \mathrm{mg} / \mathrm{ml}-4.2604 \pm 0.539261$ and $300 \mathrm{mg} /$ $\mathrm{ml}-3.3941 \pm 0.274685)$ and then gradually declined in activity (Fig. 2). However, in all the concentrations acetone extract exhibited more FRP than the ethanolic fractions. The ferric reducing power portrayed was almost at par with the ferric reducing power of ascorbic acid used as standard.

\section{Quantification of flavonoids}

Flavonoids belong to a group of natural biomolecules with variable phenolic structures available in almost 
all plant parts (Middleton 1998). They confer antioxidant property, so flavonoids present in diethyl ether, acetone, ethanol and methanol extracts were quantified and expressed as quercetin equivalent (mg QE/g). Variable quantities of flavonoids were detected in the solvent extracts (Fig. 3) with mean maximum values recorded in the ethanolic extract $(18.528 \pm 0.486124 \mathrm{mg} \mathrm{QE} / \mathrm{g})$.

\section{GC-MS analysis}

GC-MS of solvent extract in methanol revealed the presence of 39 detectable compounds in ED. The chromatogram showing characteristic peaks is represented in Fig. 4. Bioactivity of detectable compounds was studied from available literature and was observed that 22 of them had preventive and curing properties for several ailments while rests were without any reported bioactivity. Thirteen compounds like 4H-Pyran-4-one,2,3-dihydro-3,5-dihydroxy-6-methyl- (Hameed et al. 2015); 1,3,4,5-Tetrahydroxy-cyclohexanecarboxylic acid (Baratto et al. 2003; Hung et al. 2006; Matsushige et al. 1996); 3,7,11,15-Tetramethyl-2-hexadecen-1-ol (and Phytol) (Bharathy et al. 2012; Costa et al. 2016; Islam et al. 2015; Pejin et al. 2015; Rani et al. 2012; Santos et al. 2013); Hexadecanoic acid, methyl ester (Wei et al. 2011); 9,12-Octadecadienoic acid (Z,Z)-,methyl ester (Oloyede 2012); Hexadecanoic acid,2-hydroxy-1-(hydroxymethyl)ethyl ester (Tyagi and Agarwal 2017); Dioctyl phthalate (Gunalan et al. 2014); Squalene (Huang et al. 2009; Kim and

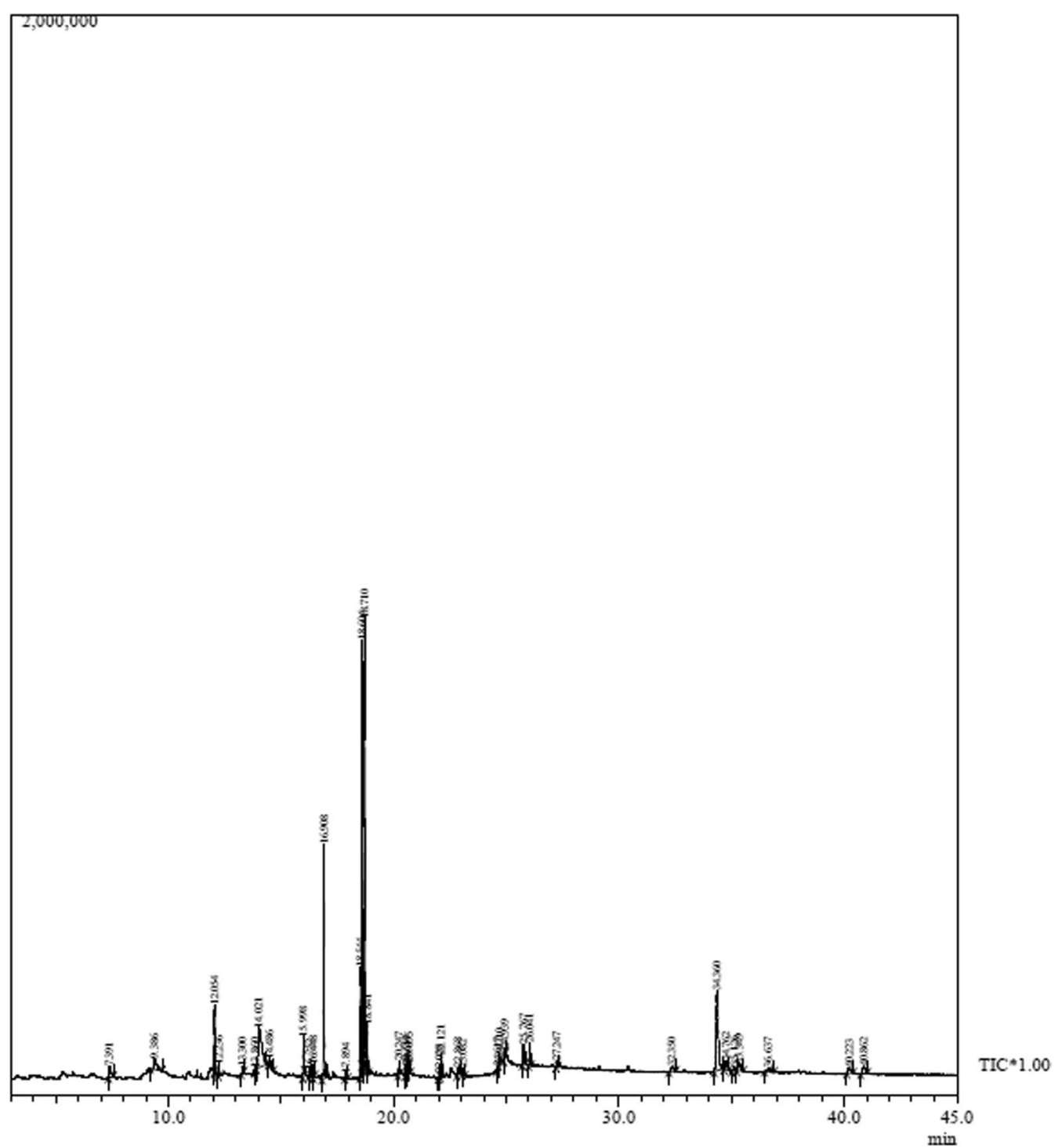

Fig. 4 GC-MS chromatogram of ED 
Karadeniz 2012; Rautela et al. 2017); Ergost-5-en-3-ol,(3. beta.,24R)- (Deora and Bano 2019; Rautela et al. 2017); Stigmast-5-en-3-ol,(3.beta.)- or ß- sitosterol (Gupta et al. 2011); Stigmasta-5,24(28)-dien-3-ol,(3.beta.)- or Fucosterol (Abdul et al. 2016); Neryl linalool isomer (Kamatou and Viljoen 2008) and D:A-Friedooleanan-7alpha-ol
(Zaidan et al. 2018) have reported antioxidant activity (Table 1). These antioxidant compounds were detected in variable amount (Fig. 5) and occupy an area of $63.92 \%$ of the total detected compounds by GC-MS. Four of these compounds, 1,3,4,5-Tetrahydroxy-cyclohexanecarboxylic acid; Hexadecanoic acid, methyl ester; Phytol and

Table 1 Chemistry of GC-MS detected antioxidant molecules

\begin{tabular}{|c|c|c|c|c|}
\hline \multirow{2}{*}{$\begin{array}{l}\text { Compounds with antioxidant activity } \\
\text { (references included in brackets) }\end{array}$} & \multicolumn{2}{|c|}{ Molecular } & \multirow[t]{2}{*}{ Retention time } & \multirow[t]{2}{*}{ Area \% } \\
\hline & Formula & $\begin{array}{l}\text { Weight } \\
\text { (g/mol) }\end{array}$ & & \\
\hline 4H-Pyran-4-one, 2,3-dihydro-3,5-dihydroxy-6-methyl- (Hameed et al. 2015) & $\mathrm{C}_{6} \mathrm{H}_{8} \mathrm{O}_{4}$ & 144.12 & 7.391 & 1.52 \\
\hline $\begin{array}{l}\text { 1,3,4,5-Tetrahydroxy-cyclohexanecarboxylic acid (Baratto et al. 2003; Hung et al. 2006; Matsushige } \\
\text { et al. 1996) }\end{array}$ & $\mathrm{C}_{7} \mathrm{H}_{12} \mathrm{O}_{6}$ & 192.17 & 14.021 & 10.80 \\
\hline \multirow{2}{*}{$\begin{array}{l}\text { 3,7,11,15-Tetramethyl-2-hexadecen-1-ol (or Phytol*) (Bharathy et al. 2012; Costa et al. 2016; Islam et al. } \\
\text { 2015; Pejin et al. 2015; Rani et al. 2012; Santos et al. 2013) }\end{array}$} & \multirow{2}{*}{$\mathrm{C}_{20} \mathrm{H}_{40} \mathrm{O}$} & \multirow[t]{2}{*}{296.5} & 16.252 & 0.37 \\
\hline & & & $18.710^{*}$ & $18.87^{*}$ \\
\hline Hexadecanoic acid, methyl ester (Wei et al. 2011) & $\mathrm{C}_{17} \mathrm{H}_{34} \mathrm{O}_{2}$ & 270.5 & 16.908 & 9.47 \\
\hline 9,12-Octadecadienoic acid (Z,Z)-, methyl ester (Oloyede 2012) & $\mathrm{C}_{19} \mathrm{H}_{34} \mathrm{O}_{2}$ & 294.5 & 18.544 & 3.54 \\
\hline Hexadecanoic acid, 2-hydroxy-1-(hydroxymethyl)ethyl ester (Tyagi and Agarwal 2017) & $\mathrm{C}_{19} \mathrm{H}_{38} \mathrm{O}_{4}$ & 330.5 & 18.604 & 15.67 \\
\hline Dioctyl phthalate (Gunalan et al. 2014) & $\mathrm{C}_{24} \mathrm{H}_{38} \mathrm{O}_{4}$ & 390.6 & 23.082 & 0.43 \\
\hline Squalene (Huang et al. 2009; Kim and Karadeniz 2012; Rautela et al. 2017) & $\mathrm{C}_{30} \mathrm{H}_{50}$ & 410.7 & 26.041 & 1.24 \\
\hline Ergost-5-en-3-ol, (3.beta.24r)- (Deora and Bano 2019; Rautela et al. 2017) & $\mathrm{C}_{28} \mathrm{H}_{48} \mathrm{O}$ & 400.7 & 32.350 & 0.82 \\
\hline Stigmast-5-en-3-ol, (3.beta.)-or sitosterol,.beta. (Gupta et al. 2011) & $\mathrm{C}_{29} \mathrm{H}_{50} \mathrm{O}$ & 414.7 & 34.360 & 12.96 \\
\hline Stigmasta-5,24(28)-dien-3-ol, (3.beta.)-or Fucosterol (Abdul et al. 2016) & $\mathrm{C}_{29} \mathrm{H}_{48} \mathrm{O}$ & 412.7 & 34.762 & 1.27 \\
\hline Neryl linalool isomer (Kamatou and Viljoen 2008) & $\mathrm{C}_{10} \mathrm{H}_{18} \mathrm{O}$ & 154.25 & 27.247 & 0.41 \\
\hline D:A-Friedooleanan-7alpha-ol (Zaidan et al. 2018) & $\mathrm{C}_{30} \mathrm{H}_{52} \mathrm{O}$ & 428.7 & 40.862 & 1.42 \\
\hline
\end{tabular}

4H-Pyran-4-one, 2,3-

dihydro-3,5-dihydroxy-6-

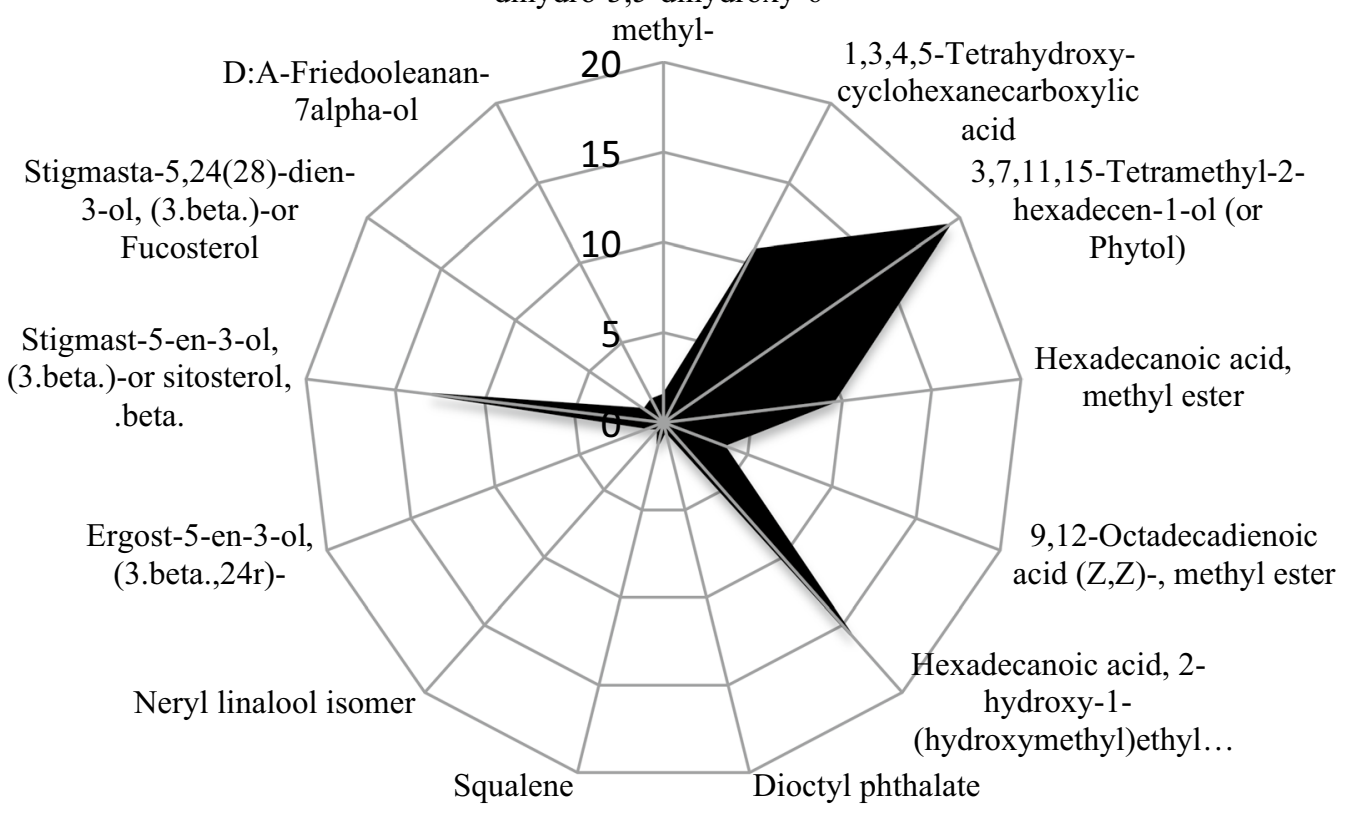

Fig. 5 Graphical representation of area percentage of GC-MS detected antioxidant molecules 
Stigmast-5-en-3-ol,(3.beta.)- or $ß$-sitosterol are present in high quantity. They cover $52.47 \%$ out of the $63.92 \%$ area occupied by the antioxidant metabolites and $82.08 \%$ area among the thirteen free radical scavengers. The antiradicals investigated in ED primarily belonged to varied chemical class, viz. fatty acid and derivatives (3); terpenoid and their steroidal counterparts (7); phenolic entities (2); and phthalate ester (1). Among these different free radical scavenging metabolites, terpenoid and its associated derivatives (58\%) form the predominant class of compounds while the lowest percentage representation is from phthalate ester (1\%) as exemplified in Fig. 6.

\section{Chromatographic separation for confirmation}

Preference of solvents for molecules is not same. So, initiative was made to chromatographically fractionalize the methanol extract with solvents like toluene, chloroform, diethyl ether, ethanol, ethanol/methanol (1:1), methanol, methanol/water (1:1) and water to execute DPPH scavenging ability for each fraction. The DPPH scavenging activity of solvent fractions showed variable results

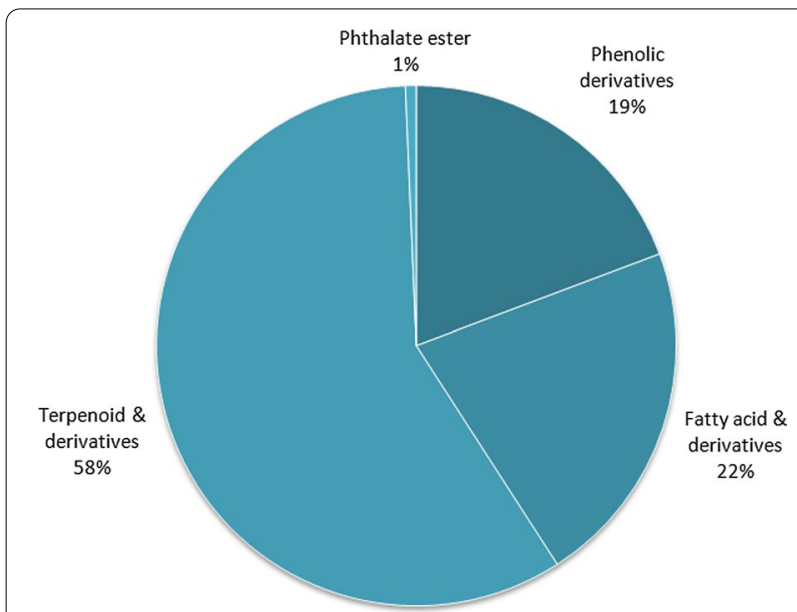

Fig. 6 Class-based pie graph of identified antioxidant compounds in $E D$ and is represented in Fig. 7a. The graph showed a major peak in DPPH scavenging activity with ethanol fraction (84.99\%).

Further sub-fractionation of ethanol fraction and collecting the sub-fractions in recurring $10 \mathrm{ml}$ volume was conducted to confirm ethanol solubility of the antioxidant molecules described previously. The results of DPPH scavenging activity of the fractions collected as recurring $10 \mathrm{ml}$ volume are depicted in Fig. $7 \mathrm{~b}$. The graph is a clear indicative of the fact that the molecules described earlier as ethanol soluble are confirmed as truly and highly soluble in ethanol as they were extracted in the first $10 \mathrm{ml}$ volume of the chromatographic fraction.

Biosynthesis pathway of potential antioxidant compounds We consider four compounds namely-1,3,4,5-Tetrahydroxy-cyclohexanecarboxylic acid (10.8\%); Hexadecanoic acid, methyl ester (9.47\%); Phytol (19.24\%); and Stigmast5-en-3-ol,(3.beta.)- or ß-sitosterol (12.96\%), present in considerably higher quantities as the chief candidates for conferring antioxidant property to the plant extract, though rest compounds also have their role in enhancing the free radical quenching activity, and altogether a cumulative effect may be concluded in respect of the remarkable antioxidant potential of ED. The pathway trends of all these potent radical quenching compounds would decipher important information regarding their site of cellular origin and molecular chemistry.

The terpenoid and their steroidal antioxidant derivatives include 7(seven) compounds, viz. Neryl linalool isomer (Linalool); 3,7,11,15-Tetramethyl-2-hexadecen-1-ol or Phytol; Squalene; Ergost-5-en-3-ol,(3.beta.,24R)- or Campesterol; Stigmast-5-en-3-ol,(3.beta.)- or ß-Sitosterol; Stigmasta-5,24(28)-dien-3-ol,(3.beta.)- or Fucosterol; and D:A-Friedooleanan-7alpha-ol or Epiputranjivol, covering an area of $58 \%$ out of the total anti-radical molecules detected, are anticipated to originate via the summative involvement of the 2-C-Methyl-D-erythritol4-phosphate/1-deoxy-D-xylulose-5-phosphate(MEP/
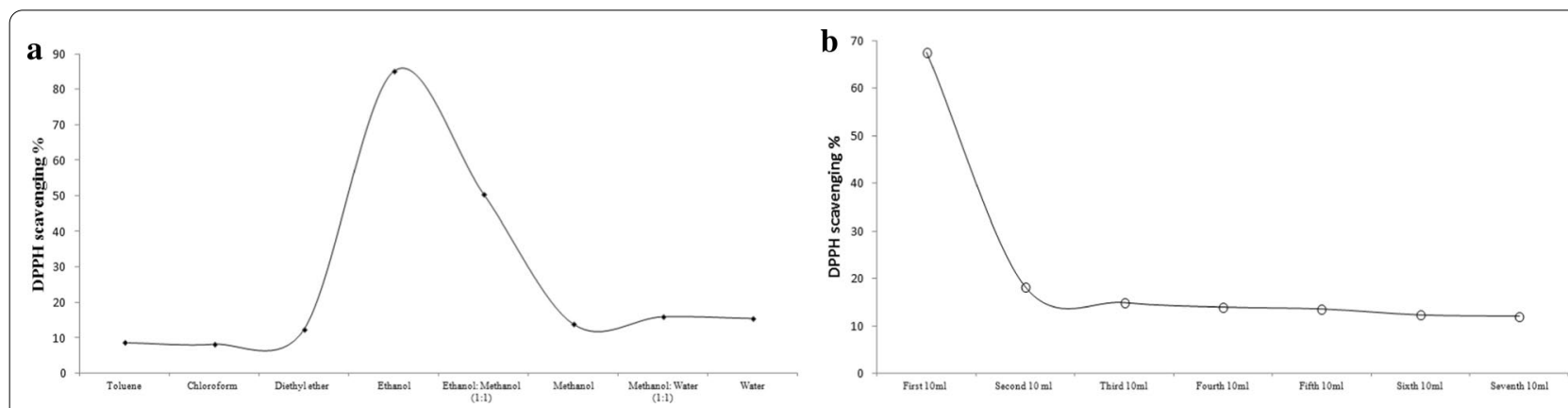

Fig. 7 Graphical representation of mean DPPH scavenging activity, a non-polar to polar solvent fractions and $\mathbf{b}$ ethanol sub-fractions 
DOXP) scheme and Mevalonic acid (MVA) pathway, exclusively and independently operating in three parallel intracellular sites: chloroplast (CP), mitochondria (MT) and cytosol with some common sets of biochemical intermediates and enzymes. Endoplasmic reticulum (ER) and peroxisomes may also be involved as a plot of occurrence of these reactions (Nagegowda 2010). Except the metabologenesis of diterpene alcohol phytol, being restricted only to the $\mathrm{CP}$ in addition to monoterpene metabolite (Linalool) being synthesized in either/both cytoplasm and $\mathrm{CP}$, the rest triterpenoid entities (Squalene) along with its phytosterolic derivatives (Epiputranjivol; Campesterol; Fucosterol; and B-Sitosterol) are simultaneously anabolized in all the three sub-cellular locations, i.e. CP, MT and cytosol, respectively.

The fatty acid(s) [FA] and their esterified counterparts (22\%) [3(three) metabolites, i.e. Hexadecanoic acid, methyl ester or methyl palmitate; 9,12-Octadecadienoic $\operatorname{acid}(\mathrm{Z}, \mathrm{Z})-$, methyl ester or methyl linoleate; and Hexadecanoic acid, 2-hydroxy-1-(hydroxymethyl) ethyl ester], are assumed to originate through the basic FA biosynthesis pathway initiating from Acetyl-CoA (A-CoA) imported from MT, with involvement of subsequent cellular organelles CP and smooth ER (SER). Post the synthesis of Palmitoyl-ACP (P-ACP)/Hexadecanoic acid (HA) in the CP, P-ACP cannot be accepted any further by the Acyl-malonyl-ACP condensing enzyme within the $\mathrm{CP}$, and therefore, extension beyond $\mathrm{C}-16$ fatty acids occur utilizing enzymes located on the cytosolic face of the SER. The desaturation reactions of corresponding long chain fatty acids, viz. Stearic acid at C-9 and 12 positions, occurs by the help of a mixed function oxidase class enzyme, EC 1.14.19.13 (fatty acyl-CoA-desaturase) generating Oleate $\left(18: 1^{\Delta 9}\right)$ and Linoleate $\left(18: 1^{\Delta 9,12}\right)$, respectively, in the SER (Nelson and Cox 2000).

The phenolic moieties (19\%) [2(two) biomolecules, namely 4H-Pyran-4-one,2,3-dihydro-3,5-dihydroxy6-methyl-; and 1,3,4,5-Tetrahydroxy-cyclohexanecarboxylic acid], too are synthesized via the Shikimic acid pathway (SP) in the CP with initial precursors Erythrose4-phosphate (E-4-P) and phosphoenol pyruvate (PEP) derived from Calvin cycle and Glycolytic pathways, respectively.

Lastly, Dioctyl phthalate [a phthalate ester(1\%)], though have been described as an antioxidant (Gunalan et al. 2014), has been excluded from any further analysis due to its uncertain origin and environmental effects (Reid et al. 2007).

\section{Discussion}

Reactive oxygen species and antioxidants have multifold roles in the life of an organism. Most of the diseases and disorders are attributed to the asymmetry in pro-oxidation and anti-oxidation reactions occurring in an organism (Bhattacharya and Chakraborty 2015). DPPH assay primarily explores the capability of plant extracts to scavenge or neutralize free radicals and thus, is considered a major experimental indicator of antioxidant activity of the extract. The percentage of recorded DPPH activity in solvent extracts showed an increase towards the polar end as the antioxidant molecules are more soluble to polar solvents. Ferric reducing power assay of acetone and ethanol reveals acetone extract to be more capable over ethanol extract. A similar type of result was observed by Labar et al. (2019) in extracts made from fresh green leaves of Camellia sinensis. Interaction and DPPH inhibition kinetics of ED extracts of acetone, ethanol and methanol further confirm DPPH scavenging potential of methanol extract. The contributor for antioxidant activity of ED was unearthed by GCMS analysis.

GC-MS analysis revealed the presence of thirteen compounds with reported antioxidant activity which must have contributed towards scavenging DPPH. Of these compounds 1,3,4,5-Tetrahydroxy-cyclohexanecarboxylic acid; Hexadecanoic acid, methyl ester; Phytol and Stigmast-5-en-3-ol, (3.beta.)-or $ß$-sitosterol are present in high quantity. 4H-Pyran-4-one, 2,3-dihydro-3,5-dihydroxy-6-methyl-; 9,12-Octadecadienoic acid (Z,Z)-, methyl ester; Squalene; and Stigmasta-5,24(28)-dien-3-ol, (3.beta.)- or Fucosterol have moderate quantitative presence while 3,7,11,15-Tetramethyl-2-hexadecen-1-ol; Hexadecanoic acid,2-hydroxy-1-(hydroxymethyl) ethyl ester; Dioctyl phthalate and Ergost-5-en-3-ol, (3.beta.,24R)- are much lower in quantity.

We tried to assess the contribution of detected antioxidant molecules in scavenging DPPH. For this, the antioxidant molecules present in extracts was grouped on the basis of their solubility. In our DPPH scavenging experiment with non-polar to polar solvent extract we had moderate activity in acetone (69.23\%) and ethanol (54.22\%) but high activity in methanol (84.64\%) extract. Compounds like 9,12-Octadecadienoic acid (Z,Z), methyl ester and Hexadecanoic acid, methyl ester are soluble in acetone; 9,12-Octadecadienoic acid (Z,Z)-, methyl ester and Phytol are soluble in ethanol while $4 \mathrm{H}-\mathrm{Pyran}-4$-one, 2,3-dihydro-3,5-dihydroxy-6-methyl-; 1,3,4,5-Tetrahydroxy-cyclohexanecarboxylic-acid; Hexadecanoic-acid, methyl-ester; Stigmast-5-en-3-ol, (3.Beta.)-or B-Sitosterol; Stigmasta-5, 24(28)-dien-3-ol,(3.Beta.)- or Fucosterol; Squalene and Ergost-5-en-3-ol, (3.Beta.,24R)- are soluble in methanol. High DPPH activity of these three organic solvents must be due to synergistic contribution of these compounds in their respective solvents. Significantly compounds like 1,3,4,5-Tetrahydroxy-cyclohexanecarboxylic acid (soluble in ethanol and methanol), 
Hexadecanoic acid, methyl ester (soluble in acetone and methanol) and Stigmast-5-en-3-ol, (3.beta.)-or sitosterol,. beta (soluble in ethanol and methanol) are present in high quantity, and thus, these three solvent extracts exhibit higher degree of DPPH scavenging activity. So, we may infer that these three compounds-1,3,4,5-Tetrahydroxycyclohexanecarboxylic acid, Hexadecanoic acid, methyl ester and Stigmast-5-en-3-ol, (3.beta.)-or ß-sitosterol, are the prime candidates conferring antioxidant potential to ED. However, we acknowledge the contribution of other antioxidant molecules present in it for providing an overall impact on antioxidant potential.

Antioxidant flavonoids and biomolecules extractable in flavonoid fraction (https://sg.inflibnet.ac.in/ jspui/bitstream/10603/148741/11/11_tables) like 4H-Pyran-4-one,2,3-dihydro-3,5-dihydroxy-6-methyl-; 1,3,4,5-Tetrahydroxy-cyclohexanecarboxylic acid; 3,7,11,15-Tetramethyl-2-hexadecen-1-ol (or Phytol); Hexadecanoic acid, methyl ester; 9,12-Octadecadienoic acid (Z,Z)-, methyl ester and Stigmast-5-en-3-ol, (3.beta.)- or $ß$-sitosterol, while other bioactive flavonoids like 1,2-Benzenedicarboxylic acid, diethyl ester and 9,12,15-Octadecatrienoic acid, methyl ester, (Z,Z,Z)took main role in quantifying the flavonoids in extract. Diethyl ether extract was quantified to have $4.064 \mathrm{mg}$ $\mathrm{QE} / \mathrm{g}$ flavonoid. This quantity is due to the presence of Hexadecanoic acid, methyl ester in high amount and 9,12-Octadecadienoic acid (Z,Z)-, methyl ester. Acetone (10.192 mg QE/g) showed higher flavonoid content due to the presence of 1,2-Benzenedicarboxylic acid, diethyl ester over Hexadecanoic acid, methyl ester and 9,12-Octadecadienoic acid (Z,Z)-, methyl ester. Ethanol (18.528 $\mathrm{mg} \mathrm{QE} / \mathrm{g}$ ) with the highest quantity of flavonoid dissolves 3,7,11,15-Tetramethyl-2-hexadecen-1-ol or Phytol and 9,12,15-Octadecatrienoic acid, methyl ester, (Z,Z,Z)- in high quantity and 9,12-Octadecadienoic acid (Z,Z)-, methyl ester in moderate amount. Methanol (2.488 mg QE/g) reported the lowest amount of flavonoid despite containing molecules like 4H-Pyran-4-one,2,3dihydro-3,5-dihydroxy-6-methyl-; Stigmast-5-en-3-ol,(3. beta.)- or $\beta$-sitosterol and Hexadecanoic acid, methyl ester. So, we can infer that besides these antioxidant and other major bioactive molecules, there must be other flavonoids that contribute towards summative effect in respect of its quantity.

Chromatographic separation of methanol extract with solvents and DPPH scavenging ability for each fraction resulted in a major peak with ethanol fraction. During the process of column chromatographic fractionation, as we expected the bioactive compounds must have been separated on the basis of their preferred solvent. Compounds like 9,12-Octadecadienoic acid (Z,Z)-, methyl ester and Phytol are soluble in ethanol. But, 9,12-Octadecadienoic acid (Z,Z)-, methyl ester is also soluble in toluene and diethyl ether, so during our process of fractionation they must have been separated by these two solvents towards the non-polar end and phytol was only left behind and accumulated in ethanol fraction. Phytol in considerable high quantity has expressed its activity as antioxidant and that has been reflected in DPPH scavenging activity. Ethanol/ methanol (1:1) contains 4H-Pyran-4-one, 2,3-dihydro-3,5-dihydroxy-6-methyl-; 1,3,4,5-Tetrahydroxy-cyclohexanecarboxylic acid; Stigmast-5-en-3-ol, (3.Beta.)-or ß-Sitosterol; Stigmasta-5,24(28)-dien3-ol,(3.Beta.)- or Fucosterol; Squalene; Ergost-5-en-3-ol, (3.Beta.,24R)-, so its DPPH scavenging activity is due to synergistic activity of all these compounds. Hexadecanoic acid, methyl ester though soluble in methanol gets separated by diethyl ether towards the non-polar end. Finally, trace amount of bioactive compounds are left behind to show their potential in following methanol, methanol/ water (1:1) and water fractions.

The antioxidant pathway analysis deduced three separate metabologenesis schemes to explain the origin of these varied antioxidant molecules in ED. Figure 8 represents the summative pathway diagram representing the biosynthesis of these antioxidant compounds. Terpenoid molecules originate via the MEP/DOXP or MVA pathway; FA and their derivatives are anabolized through the FA biosynthesis pathway and Phenolic counterparts are synthesized by the SP.

The MVA pathway initiates in the cytosol with A-CoA being the initial metabolite channelled from the MT and successively transformed to 3-Hydroxy-3-Methylglutaryl-CoA (HMG-CoA). It is converted to MVA by the enzyme HMG-CoA reductase (1.1.1.34) [HMGR], an important enzyme regulating the synthesis of MVA and its corresponding downstream metabolites (Nagegowda 2010). MVA is modified to Isopentyl phosphate that serves as the immediate precursor in the synthesis of Isopentyl pyrophosphate (IPP). The MEP/DOXP pathway initiates with glycolytic terminal product pyruvate combining with Erythrose-4-phosphate in the CP, to synthesize 1-Deoxy-D-xylulose-5-phosphate; which through a series of reactions yields (E)-4-Hydroxy-3-methylbut2-enyl-diphosphate (HMBPP). HMBPP serves as the common precursor in the production of IPP and Dimethylallyl pyrophosphate (DMAPP). IPP and DMAPP are inter-convertible isomers of each other and their metabolic equilibrium is maintained by the enzyme Isopentyl diphosphate isomerase (IDI), an important rate determining enzyme of the terpenoid backbone biosynthesis scheme (Nagegowda 2010). One molecule each of IPP and DMAPP combines with each other to synthesize Geranyl pyrophosphate (GPP), which on addition of another molecule of GPP produces geranyl geranyl pyrophosphate 


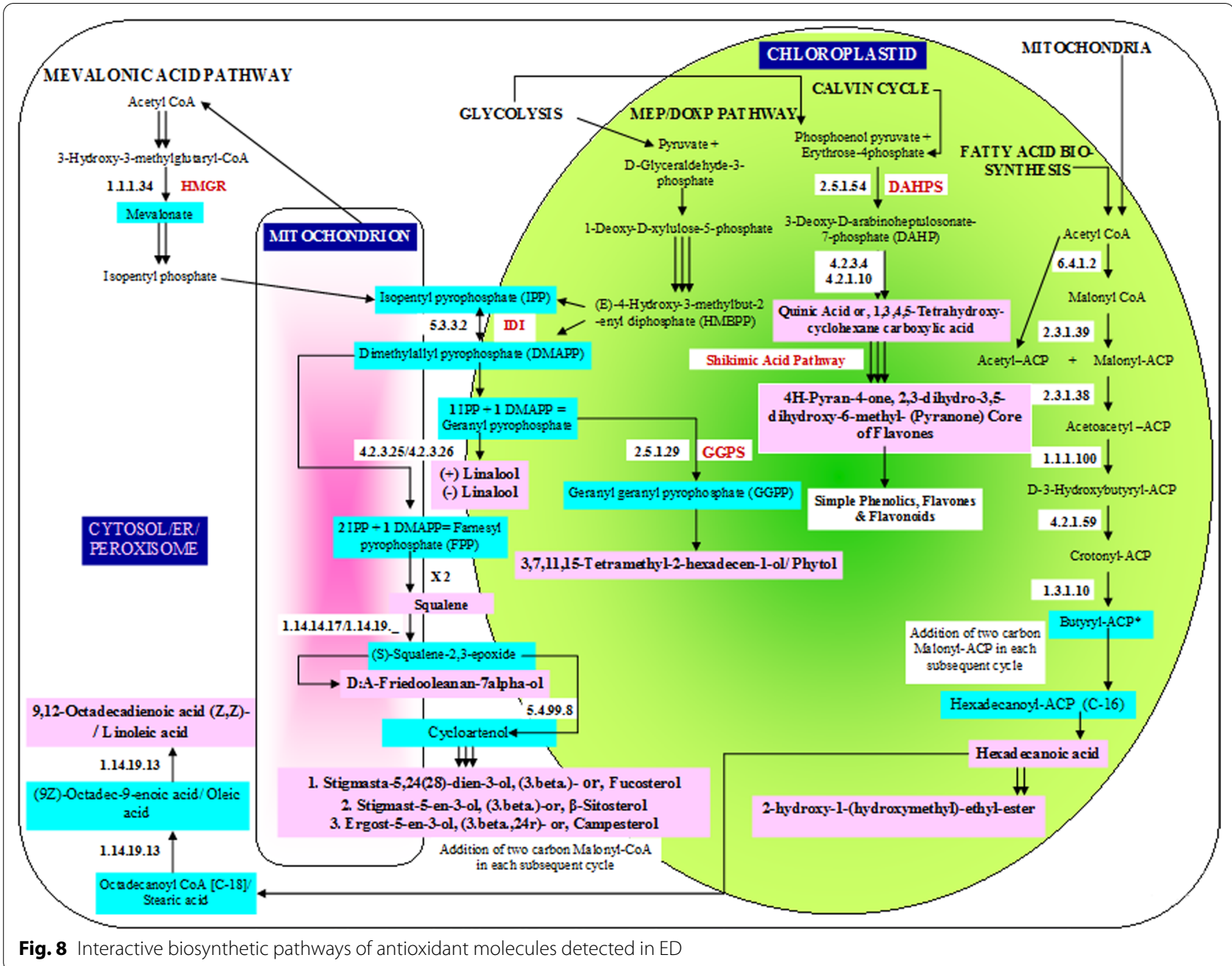

(GGPP). GPP ultimately gives to monoterpenoid metabolite Linalool $\left(\mathrm{C}_{10} \mathrm{H}_{18} \mathrm{O}\right)$, a tertiary alcohol which is an octa-1,6-diene substituted by methyl groups at positions 3,7 and a hydroxy group at position 3 while GGPP synthesizes 3,7,11,15-Tetramethyl-2-hexadecen-1-ol or Phytol $\left(\mathrm{C}_{20} \mathrm{H}_{40} \mathrm{O}\right)$, an acyclic diterpene alcohol and a constituent of chlorophyll ubiquitous in all members of Plantae in the presence of the enzyme geranyl geranyl diphosphate synthase (GGPS) [EC 2.5.1.29], another rate limiting enzyme of the terpenoid metabologenesis corridor (Nagegowda 2010). Two molecules of IPP and one molecule of DMAPP add up to generate Farnesyl pyrophosphate (FPP). Two molecules of FPP subsequently condense to synthesize Squalene $\left(\mathrm{C}_{30} \mathrm{H}_{50}\right)$. Squalene is a triterpene and is a precursor for synthesis of all plant and animal sterols. Squalene is further converted to (S)Squalene-2,3-epoxide by the enzyme Squalene monooxygenase (EC 1.14.14.17). This 2,3-Oxidosqualene gives rise to D:A-Friedooleanan-7-ol,(7.Alpha.)- $\left[\mathrm{C}_{30} \mathrm{H}_{52} \mathrm{O}\right]$ or Epiputranjivol; and Cycloartenol in two separate reactions. The conversion of (S)-Squalene-2,3-epoxide to Cycloartenol is accomplished in the presence of the biocatalyst Cycloartenol synthase (EC 5.4.99.8). Cycloartenol serves as a common metabolic precursor in the biosynthesis of the three sterolic triterpenoid antioxidants, viz. Fucosterol $\left(\mathrm{C}_{29} \mathrm{H}_{48} \mathrm{O}\right)$, $\beta$-Sitosterol $\left(\mathrm{C}_{29} \mathrm{H}_{50} \mathrm{O}\right)$ and Campesterol $\left(\mathrm{C}_{28} \mathrm{H}_{48} \mathrm{O}\right)$, respectively.

HA-methyl ester $\left(\mathrm{C}_{17} \mathrm{H}_{34} \mathrm{O}_{2}\right)$ commonly known as methyl palmitate belongs to the class of organic compounds called fatty acid methyl ester. This saturated fatty acid is commonly found in plants, animals and microorganisms. Synthesis of HA initiates in the $\mathrm{CP}$ with $\mathrm{A}-\mathrm{CoA}$ as raw material being imported from the Tri-Carboxylic acid (TCA) cycle occurring in MT and proceeds through the FA biosynthesis pathway with Malonyl-CoA (M-CoA) as the first synthesized metabolic intermediate; by the enzyme A-CoA carboxylase (EC 6.4.1.2) attributed to be main enzyme controlling the fatty acid metabologenesis scheme (Ohlrogge and Jaworski 1997). The M-CoA produced is transformed to 
Malonyl-ACP (M-ACP) in the presence of biocatalyst Malonyl transacylase (EC 2.3.1.39), which further reacts with Acetyl-ACP [a modified A-CoA biomolecule aided by the enzyme A-CoA transacylase (EC 2.3.1.38)] to generate Acetoacetyl-ACP (Ac-Ac-ACP). The Ac-Ac-ACP is subsequently converted to D-3-Hydroxybutyryl-ACP (D-3-H-ACP) by the enzyme $\beta$-Ketoacyl-ACP reductase (EC 1.1.1.100). This reaction is followed by the successive conversion of D-3-H-ACP to Crotonyl-ACP in the presence of biocatalyst 3-Hydroxyacyl-ACP dehydratase (EC 4.2.1.59) which is finally transformed to terminal metabolite Butyryl-ACP by enzyme Enoyl-ACP reductase [EC 1.3.1.10]. This reaction cycle is being repeated with M-ACP adding 2-Carbon units in every subsequent round until the formation of P-ACP (16-Carbon compound) followed by a terminal hydrolysis reaction aided by an enzyme Thioesterase (EC 3.1.2.14) to ultimately yield HA. 2-hydroxy-1-(hydroxymethyl)-ethyl-ester commonly regarded as 2-palmitoyl glycerol $\left(\mathrm{C}_{19} \mathrm{H}_{38} \mathrm{O}_{4}\right)$, too is biosynthesized/derived from HA. It is a $\beta$-monoacyl glycerol and a 2-monoglyceride where the acyl group is hexadecanoyl (palmitoyl). Further, addition of 2-Carbon $\mathrm{M}-\mathrm{CoA}$ to HA produces Octadecanoyl-CoA/Stearic acid in SER, which post twice successive rounds of desaturation synthesize Linoleic acid, an essential FA, identified as a methyl esterified derivative in ED owing to methanolic leaf extract being used for GC-MS analysis. This metabolite Methyl linolenate or 9,12-Octadecadienoic $\operatorname{acid}(Z, Z)$-, methyl ester $\left(\mathrm{C}_{19} \mathrm{H}_{34} \mathrm{O}_{2}\right)$, is an ester of omega- 6 polyunsaturated fatty acid (PUFA), attributed to be a powerful antioxidant molecule.

1,3,4,5-Tetrahydroxy-cyclohexane-carboxylic acid $\left(\mathrm{C}_{7} \mathrm{H}_{12} \mathrm{O}_{6}\right)$, commonly known as Quinic acid or Kinic acid, is a conjugate acid of quinate. Chemically, it is a cyclitol, a cyclic polyol, and a cyclohexanecarboxylic acid synthesized by the SP occurring in the CP. Even 4H-Pyran-4-one,2,3-dihydro-3,5-dihydroxy-6-methyl- or Pyranone $\left(\mathrm{C}_{6} \mathrm{H}_{8} \mathrm{O}_{4}\right)$ too follow the footsteps of the same $\mathrm{SP}$ and is synthesized as a subsidiary product in the synthesis of simple phenolics and flavone moieties in ED. The initial precursors involved in SP, i.e. PEP and E-4-P, reacts to generate 3-Deoxy-D-arabino-heptulosonate7-phosphate (DAHP) aided by the enzyme DAHP synthase. DAHP synthase (EC 2.5.1.54), a transferase class enzyme, may be attributed to be the chief initial biocatalyst controlling the metabolic outputs of the SP, as it regulates the gateway leading to synthesis of the first Shikimate scheme intermediate DAHP (Tzin et al. 2012). This DAHP is further converted to 3-Dehydroquinate (3-DHQ) in the presence of EC 4.2.3.4 (3-DHQ synthase) which post a dehydrogenation reaction (probable enzyme EC 4.2.1.10/3-DHQ dehydratase) yields Quinic acid.
The above-described independent pathways operate parallelly and cumulatively affecting the metabologenesis of these varied classes of antioxidant metabolites which confers this remarkable free radical scavenging potential to ED plant extract.

\section{Conclusion}

Antioxidants have diverse role to play in the life of an organism as major diseases and disorders are mainly attributed to the imbalance between pro-oxidation and antioxidation reactions occurring in the living system. Edgaria darjeelingensis is a treasure of antioxidant molecules of which some of them are in significant quantities. Isolation of these molecules for pharmaceutical industry can be a boon for mankind.

\section{Abbreviations}

ED: Edgaria darjeelingensis; GC-MS: Gas chromatography mass spectrometry; DPPH: 2,2-Diphenyl-1-picrylhydrazyl; AE: Ascorbic acid equivalent; QE: Quercetin equivalent; FRP: Ferric reducing power; MEP: 2-C-Methyl-D-erythritol4-phosphate; DOXP: 1-Deoxy-D-xylulose-5-phosphate; MVA: Mevalonic acid/ mevalonate; CP: Chloroplast; MT: Mitochondria; ER: Endoplasmic reticulum; FA: Fatty acid(s); A-CoA: Acetyl-CoA; SER: Smooth endoplasmic reticulum; P-ACP: Palmitoyl-ACP; HA: Hexadecanoic acid; SP: Shikimic acid pathway; E-4-P: Erythrose-4-phosphate; PEP: Phosphoenol pyruvate; HMG-CoA: 3-Hydroxy-3-methylglutaryl-CoA; HMGR: HMG-CoA reductase; IPP: Isopentyl pyrophosphate; HMBPP: (E)-4-Hydroxy-3-methylbut-2-enyl-diphosphate; DMAPP: Dimethylallyl pyrophosphate; IDI: Isopentyl diphosphate isomerase; GGPP: Geranyl geranyl pyrophosphate; GPP: Geranyl pyrophosphate; GGPS: Geranyl geranyl diphosphate synthase; FPP: Farnesyl pyrophosphate; TCA: Tri-carboxylic acid; M-CoA: Malonyl-CoA; M-ACP: Malonyl-ACP; Ac-AC-ACP: Acetoacetyl-ACP; D-3-H-ACP: D-3-Hydroxybutyryl-ACP; PUFA: Polyunsaturated fatty acid; DAHP: 3-Deoxy-Darabino-heptulosonate-7-phosphate; 3-DHQ: 3-Dehydroquinate.

\section{Acknowledgements}

The authors would like to acknowledge Dr. Ashoke Bhattacharya, Senior Taxonomist, Department of Botany, Darjeeling Government College, Darjeeling, for identifying the plant and extending help during collection.

\section{Authors' contributions}

SC and MB conceived the idea. SC, SM, AG and SS carried out collection of plant material and wet laboratory experiments. SC analysed the results and wrote the draft manuscript. All authors have read and approved the manuscript.

\section{Funding}

Not applicable.

\section{Availability of data and materials}

All data generated and analysed during this study are included in this article and preserved for future reference.

Ethics approval and consent to participate

Not applicable.

\section{Consent for publication}

Not applicable.

\section{Competing interests}

The authors declare that they have no competing interest.

Received: 10 November 2020 Accepted: 14 February 2021 Published online: 25 February 2021 


\section{References}

Abdul QA, Choi RJ, Jung HA, Choi JS (2016) Health benefit of fucosterol from marine algae: a review. J Sci Food Agric 96(6):1856-1866

Baratto MC, Tattini M, Galardi C, Pinelli P, Romani A et al (2003) Antioxidant activity of galloylquinic derivatives isolated from Pistacia lentiscus leaves. Free Radic Res 37(4):405-412

Bharathy $V$, Sumathy BM, Uthayakumari F (2012) Determination of phytocomponents by GC-MS in leaves of Jatropha gossypifolia L. Sci Res Repot 2(3):286-290

Bhattacharya M, Chakraborty S (2015) Free radicals and naturally occurring antioxidants. J Pharmacogn Phytochem 3:1-7

Bhattacharya M, Mandal P, Sen A (2009) In vitro detection of antioxidants in different solvent fractions of Ginger (Zingiber officinale Rosc.). Indian J Plant Physiol 14(1):23-27

Costa P, Islam J, Santos TS, Ferreira PB, Oliveira LS et al (2016) Evaluation of antioxidant activity of phytol using non- and pre-clinical models. Curr Pharm Biotechnol 17(14):1278-1284

Deora GS, Bano I (2019) Preliminary phytochemical screening and GC-MS analysis of methanolic leaf extract of Abutilon pannosum (Forst F.) Schlect. from Indian Thar desert. J Pharmacogn Phytochem 8(1):894-899

Gunalan G, Krishnamurthy V, Saraswathy A (2014) GC-MS and HPTLC fingerprinting of Bauhinia variegata leaves for anticancer activity. World J Pharm Res 3:1313-1336

Gupta R, Sharma AK, Dobhal MP, Sharma MC, Gupta RS (2011) Antidiabetic and antioxidant potential of $\beta$-sitosterol in streptozotocin induced experimental hyperglycemia. J Diabetes 3(9):29-37

Hameed IH, Hussein HJ, Kareem MA, Hamad NS (2015) Identification of five newly described bioactive chemical compounds in methanolic extract of Mentha viridis by using gas chromatography-mass spectrometry (GC-MS). J Pharmacognosy Phytother 7(7):107-125

Huang ZR, Lin YK, Fang JY (2009) Biological and pharmacological activities of squalene and related compounds: potential uses in cosmetic dermatology. Molecules 14(1):540-554

Hung TM, Na M, Thuong PT, Su ND, Sok D et al (2006) Antioxidant activity of caffeoylquinic acid derivatives from the roots of Dipsacus asper Wall. J Ethnopharmacol 108(2):188-192

Islam MT, de Alencar MVOB, da Conceição MK, da Conceição MK, de Carvalho Melo-Cavalcante AA, de Sousa DP, de Freitas RM (2015) Phytol in a pharma-medico-stance. Chem Biol Interact 240:60-73

Kamatou GPP, Viljoen AM (2008) Linalool-a review of a biologically active compound of commercial importance. Nat Prod Commun. https://doi. org/10.1177/1934578X0800300727

Kim SK, Karadeniz F (2012) Biological importance and applications of squalene. Adv Food Nutr Res 65:223-233.

Labar R, Sarkar I, Sen A, Bhattacharya M (2019) Effect of solvent with varying polarities on phytochemical extraction from mature tea leaves and its evaluation using biochemical, antimicrobial and in silico approaches. Int Res J Pharm 10(8):59-67

Matsushige K, Basnet P, Kadota S, Namba T (1996) Potent free radical scavenging activity of dicaffeoylquinic acid derivatives from propolis. J Trad Med 13:217-228

Majumder S, Ghosh A, Bhattacharya M (2020) Natural anti-inflammatory terpenoids in Camellia japonica leaf and probable biosynthesis pathways of the metabolome. Bull Natl Res Cent (Egypt) 44(1):1-4
Middleton E (1998) Effect of plant flavonoids on immune and inflammatory cell function. Flavonoids in the living system. Springer, Boston, pp 175-182

Mueller-Boeker U (1993) Ethnobotanical studies among the Chitawan Tharus. J Nepal Res Cent 9:17-56

Nagegowda DA (2010) Plant volatile terpenoid metabolism: biosynthetic genes, transcriptional regulation and subcellular compartmentation. FEBS Lett 584(14):2965-2973

Nelson DL, Cox MM (2000) Lehninger principles of biochemistry. Worth Publishers, NY

Ohlrogge JB, Jaworski JG (1997) Regulation of fatty acid synthesis. Annu Rev Plant Biol 48(1):109-136

Oloyede GK (2012) Antioxidant activities of methyl ricinoleate and ricinoleic acid dominated Ricinus communis seeds extract using lipid peroxidation and free radical scavenging methods. Res J Med Plant 6(7):511-520

Pejin B, Ciric A, Glamoclija J, Nikolic M, Sokovic M (2015) In vitro anti-quorum sensing activity of phytol. Nat Prod Res 29(4):374-377

Rani JMJ, Chandramohan G, Renganathan R (2012) Antioxidant activity, preliminary phytochemical investigation and GCMS study of Bougainvillea Choicy. leaves. Int J Pharm 4(S2):12-16

Rautela I, Sharma MD, Sharma N, Kishor K, Singh K, Sharma N (2017) Comparative GC-MS analysis of leaf and root extract of medicinal plant Withania somnifera. World J Pharm Res 7:956-972

Reid AM, Brougham CA, Fogarty AM, Roche JJ (2007) An investigation into possible sources of phthalate contamination in the environmental analytical laboratory. Int J Environ An Ch 87(2):125-133

Santos CCDMP, Salvadori MS, Mota VG, Costa LM, de Almeida AAC et al (2013) Antinociceptive and antioxidant activities of phytol in vivo and in vitro models. Neuroscience. https://doi.org/10.1155/2013/949452

Shrestha I, Khadgi P (2019) Herbal veterinary practices by Tamang community in central Nepal. NUTA J6(1-2):5-11

Tyagi T, Agarwal M (2017) Phytochemical screening and GC-MS analysis of bioactive constituents in the ethanolic extract of Pistia stratiotes L. and Eichhornia crassipes (Mart.) Solms. J Pharmacogn Phytochem 6(1):195-206

Tzin V, Galili G, Aharoni A (2012) Shikimate pathway and aromatic amino acid biosynthesis. eLS. https://doi.org/10.1002/9780470015902.a0001315. pub2

Wei LS, Wee W, Fu Siong JY, Syamsumir DF (2011) Characterization of anticancer, antimicrobial, antioxidant properties and chemical compositions of Peperomia pellucida leaf extract. Acta Med Iran 49:670-674

Zaidan UH, Hamid SNM, Yusof MFM, Ahmad S, Gani SSA, Shamsi S (2018) Chemical evaluation and antioxidant properties of extracts and essential oil from Stevia rebaudiana leaves. Malays Appl Biol 47:15-23

Zou Y, Lu Y, Wei D (2004) Antioxidant activity of a flavonoid-rich extract of Hypericum perforatum L. in vitro. J Agric Food Chem 52(16):5032-5039

\section{Publisher's Note}

Springer Nature remains neutral with regard to jurisdictional claims in published maps and institutional affiliations.

\section{Submit your manuscript to a SpringerOpen ${ }^{\circ}$ journal and benefit from:}

- Convenient online submission

- Rigorous peer review

- Open access: articles freely available online

- High visibility within the field

- Retaining the copyright to your article

Submit your next manuscript at springeropen.com 